\title{
las estructuras mixtas de hormigón y aceno
}

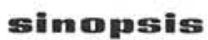

Se ponen de relieve las principales causas de la poca difusión de las estructuras mixtas en el campo de la construcción española ac. tual, y la posibilidad de que, en un futuro próximo, esta técnica llegue a utilizarse sin reservas en los casos que su empleo sea indicado. Muy sucintamente se presentan un método sencillo aproximado y otro más preciso debido a Sattler, para el cálculo de secciones. Se muestra también, mediante un ejemplo, la utilización de unas tablas y un programa de cálculo electronico que han de figurar en una miento de este tipo de estructuras.
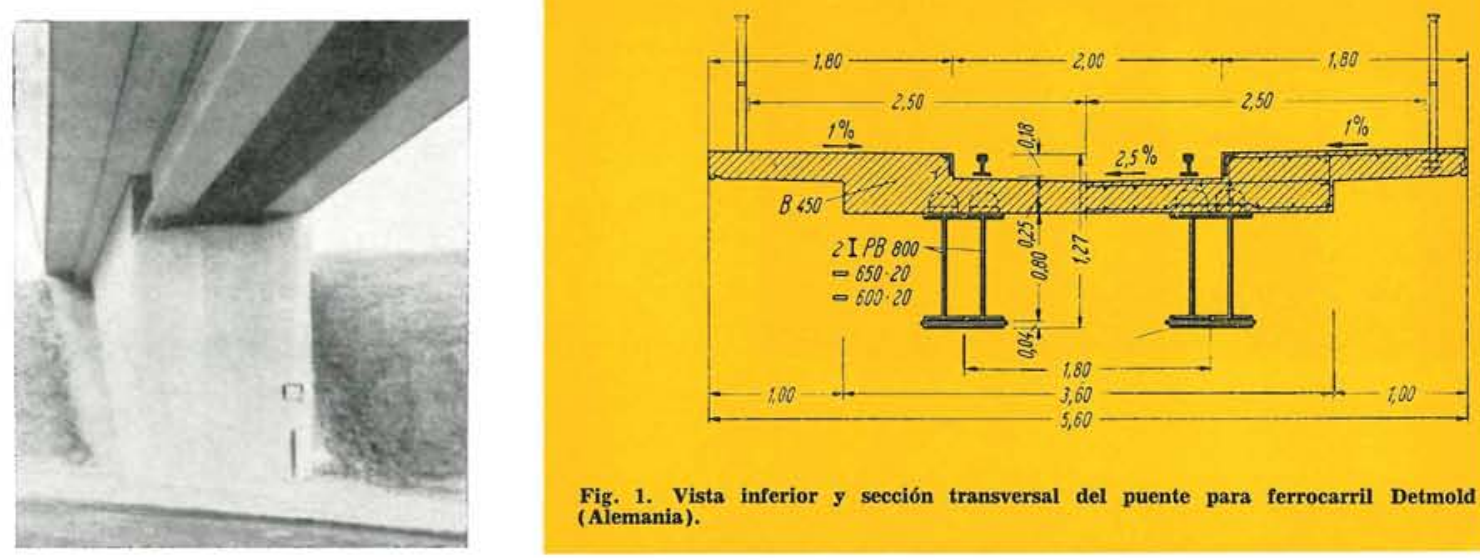

Fig. 1. Vista inferior $y$ sección transversal del puente para ferrocarril Detmold (Alemania).

En el panorama general del cálculo y diseño de estructuras existente hoy día en España, puede apreciarse - a poco que el tema se analice-la falta, o bien la escasa difusión, de determinados sistemas y procesos constructivos que en otros países se utilizan en forma mucho más profusa. El presente artículo pretende fijar la atención sobre un tema que responde netamente a estas características de abandono citadas: las estructuras mixtas de hormigón y acero.

En casi todo de cuanto sucede existe una lógica, una forma de pensar, que explica el porqué o el por qué no, como en este caso, de un proceso, siendo el fenómeno casual-por extraño que éste sea-bastante improbable.

Parece sencillo de determinar como causas principales-reales o aparentes-de la no utilización por los técnicos del campo de las estructuras mixtas, las siguientes:

Escasa o nula información; tanto teórica como práctica.

Dificultad de cálculo; que más bien cabría denominar laboriosidad, puesto que, salvo en casos de importancia, la base teórica precisa es muy reducida.

Técnica posteriormente desarrollada; los primeros trabajos comienzan en Estados Unidos y Alemania hacia el año 1940.

Por supuesto, estas tres causas no pueden aislarse, sino que se han producido ligadas entre sí, y con otras mucho más pequeñas y difíciles de estimar, cuáles podrían ser: campo de aplicaciones aparentemente reducido; escaso número de técnicos que dominen por igual las estructuras metálicas y de hormigón; etc... 

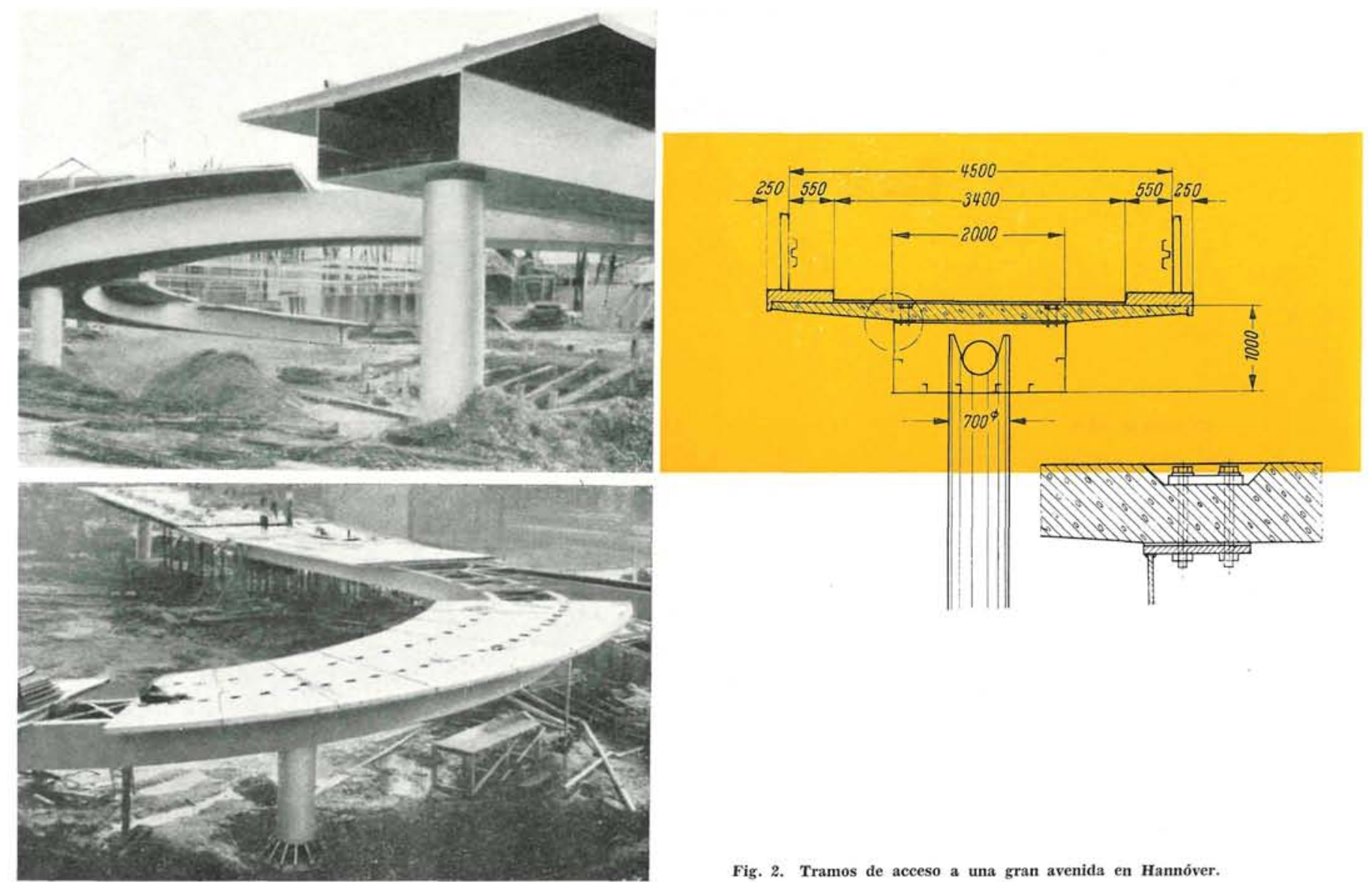

Fig. 2. Tramos de acceso a una gran avenida en Hannóver.

Sin embargo, no parece aventurado afirmar que este sistema constructivo ha de irse introduciendo poco a poco en el conjunto de disponibilidades del técnico-no desplazando a ningún otro, pero sí recabando la atención cuando el problema funcional o económico del caso patentice su empleo-, puesto que los factores que dificultaban su utilización han de ir poco a poco desapareciendo, y las ventajas constructivas que el sistema brinda van paulatinamente ofreciendo mayor interés.

Así, el cálculo electrónico ha eliminado una de las posibles dificultades; la mayor técnica constructiva - el paso más lento, quizá-permitirá su empleo con todas sus grandes posibilidades; y, fundamentalmente, los ahorros en mano de obra, materiales y plazos de ejecución, están comenzando a poseer una importancia económica sensiblemente apreciable.

Con respecto a la información, y pensando en todas estas cosas, el autor de este artículo, hace poco más de dos años, pretendió recopilar los datos más importantes del sistema, en las realizaciones alemanas y americanas-países en que primero adquirió importancia este tipo de construcción-, dando lugar a una monografía más o menos útil. Esta primera idea se vio superada, y lo que va a salir a la luz muy próximamente es bastante más que una simple puesta al día, ya que se tratará de dar una visión casi completa del sistema estructural y sus especiales características (Ref. 1).

La idea de este artículo y un siguiente, pretenden estar también en la línea informativa y son tan sólo una somera exposición del tema al técnico que, o bien conoce el sistema muy superficialmente, inclusive utilizado por él con enormes reservas, o bien prácticamente lo ignora-situación bastante usual-, remitiendo a la antedicha publicación, de la cual esto es un pequeño extracto, o bien a las fuentes de ambos (Refs. 2 y 3 ).

(Ref. 1): J. Martínez Calzón: «Estructuras mixtas. Teoría y Práctica».

(Ref. 2): K. Sattler: "Theorie der Verbundkonstruktionen".

(Ref. 3): B. Fritz: «Verbundträger. Berechunngsverfahren für die Brückenbaupraxis». 

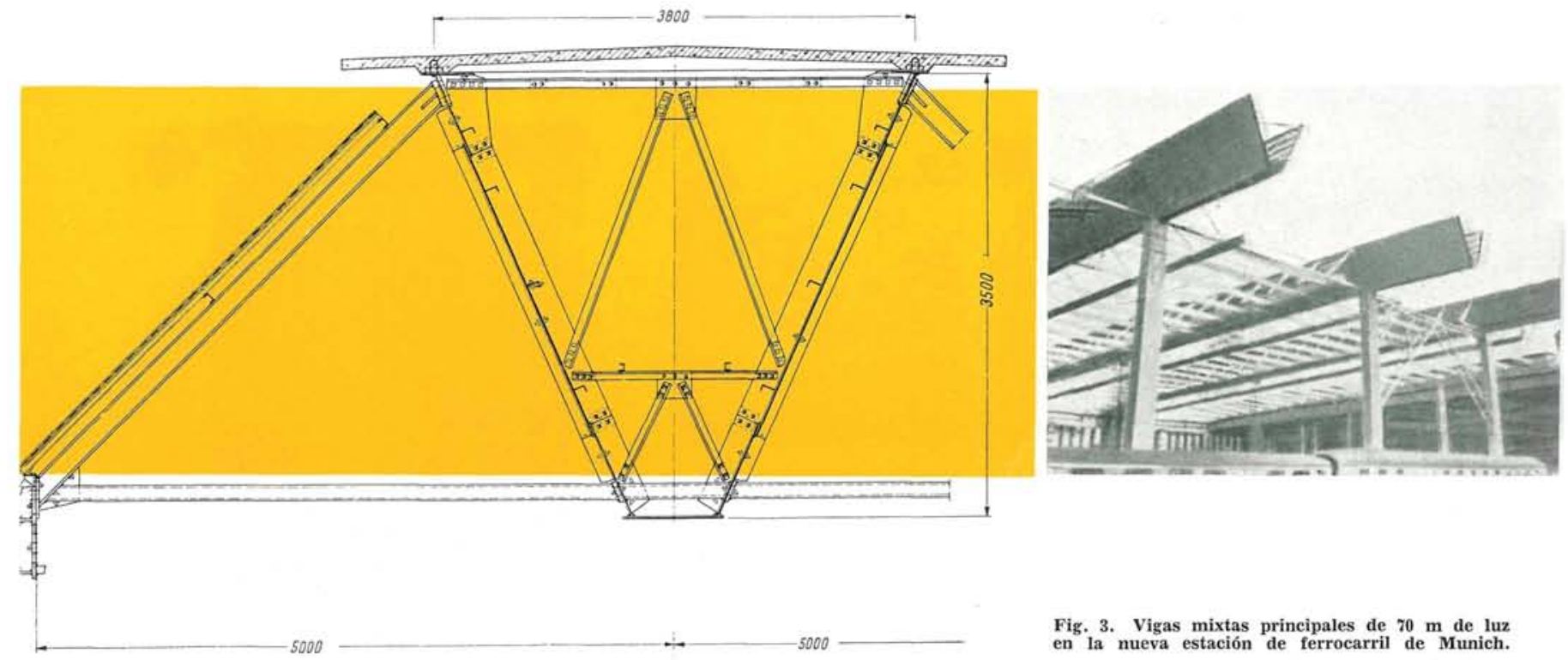

Fig. 3. Vigas mixtas principales de $70 \mathrm{~m}$ de luz en la nueva estación de ferrocarril de Munich.

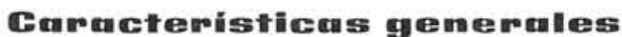

La utilización de los materiales en forma y función adecuadas a sus características es una constante de la construcción desde sus primeros pasos como técnica, una vez abandonado el camino de la artesanía y la intuición, y es una necesidad de todos los sistemas actuales, puesto que de otra forma, antes o después, perderían el interés que una coyuntura particular puede proporcionar.

La estructura mixta nace al intentar obtener las máximas ventajas que pueden ofrecer los dos materiales que la forman. El acero: lineal; resistente netamente a tracción; prefabricado totalmente. El hormigón: formáceo; económicamente resistente a compresión; inercia, asimismo económica. Estas características están también presentes en las técnicas del hormigón armado y pretensado-y han permitido realizar obras excepcionales-; sin embargo, estos sistemas constructivos se diferencian netamente de los sistemas mixtos en muy diversos puntos: forma de construcción; eliminación casi total del hormigón en tracción, incluso en los estados próximos a rotura; diferente forma de unión de los materiales; distinta colaboración frente a los esfuerzos transversales; etc.

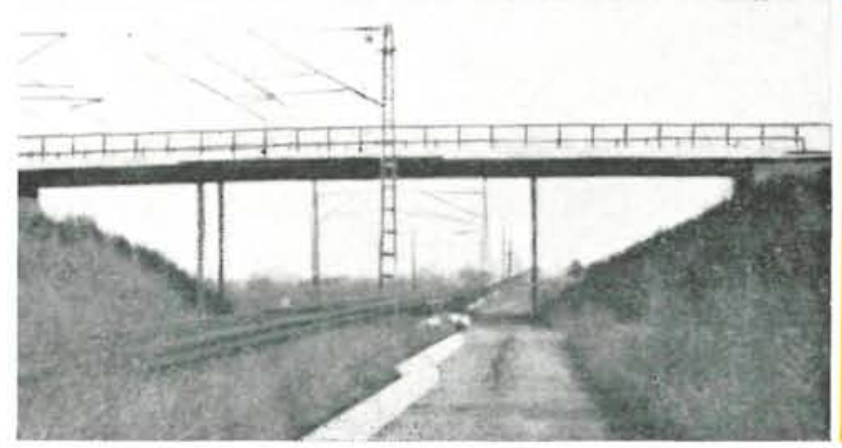

Fig. 4. Paso superior en esviaje en Roxheim (Alemania).

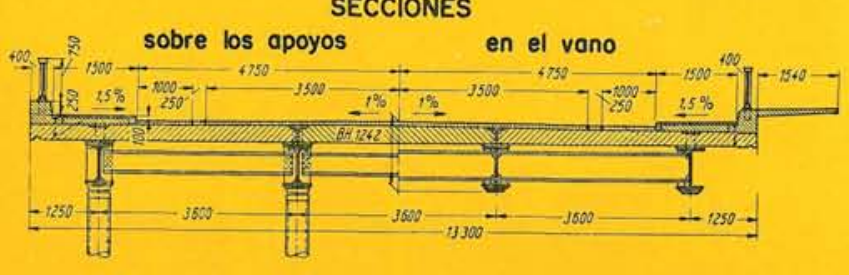

101 


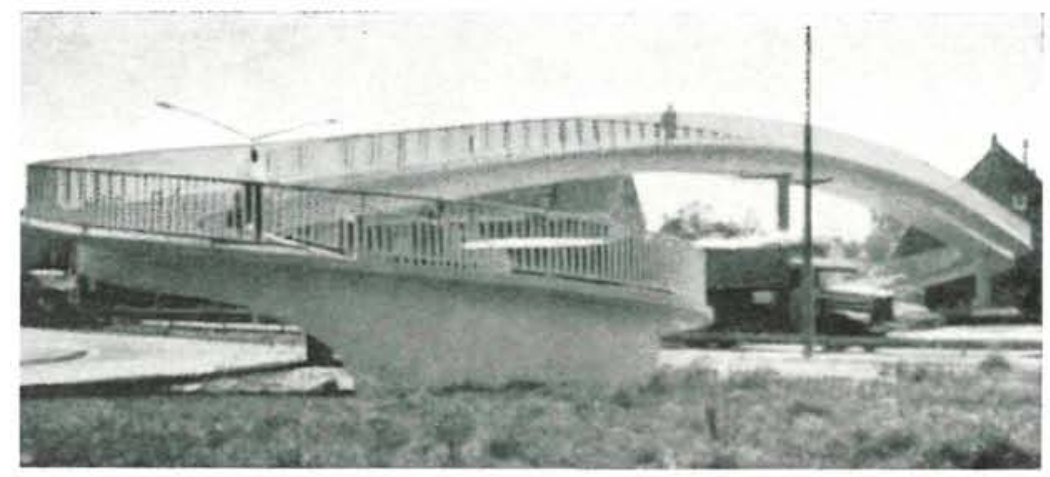

En los casos en que el sistema mixto se adapte a las necesidades de un determinado caso, las diferencias antedichas se traducen en ventajas de diverso tipo. Así, con respecto a las estructuras de hormigón armado, pueden citarse: la disminución de cantos y pesos propios, provenientes del aumento de rigidez de la sección y de la eliminación del material innecesario; la disminución de encofrados, mano de obra y elementos constructivos. Y con respecto a las estructuras metálicas: notable disminución del material, motivada por el aprovechamiento de la capacidad resistente de los elementos superficiales, necesarios para el uso de la estructura o para resistir los esfuerzos en dirección ortogonal, y que, en general, han de colocarse independientemente del tipo estructural elegido; mayor ligazón y rigidez de conjunto de la estructura.

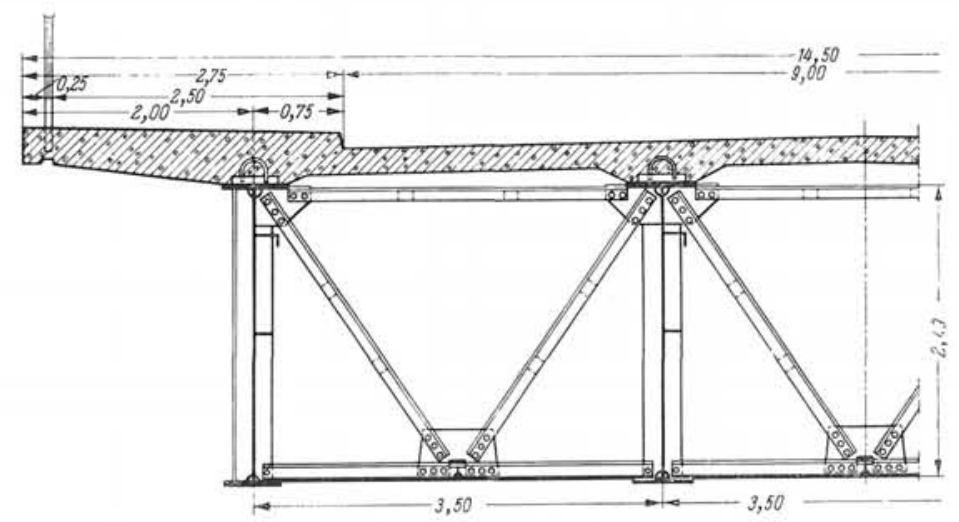

Fig. 5. Pasarela para peatones en Essen, con luces de 27 metros.
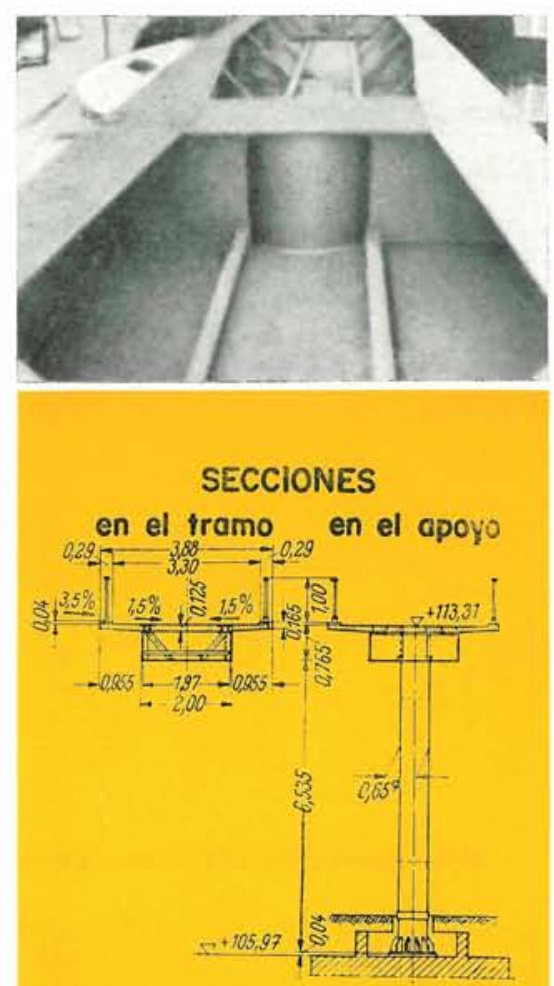

Fig. 6. Puente sobre el Drau en Villach Fig. 6. Puente
(Alemaina), con $80 \mathrm{~m}$ de luz.

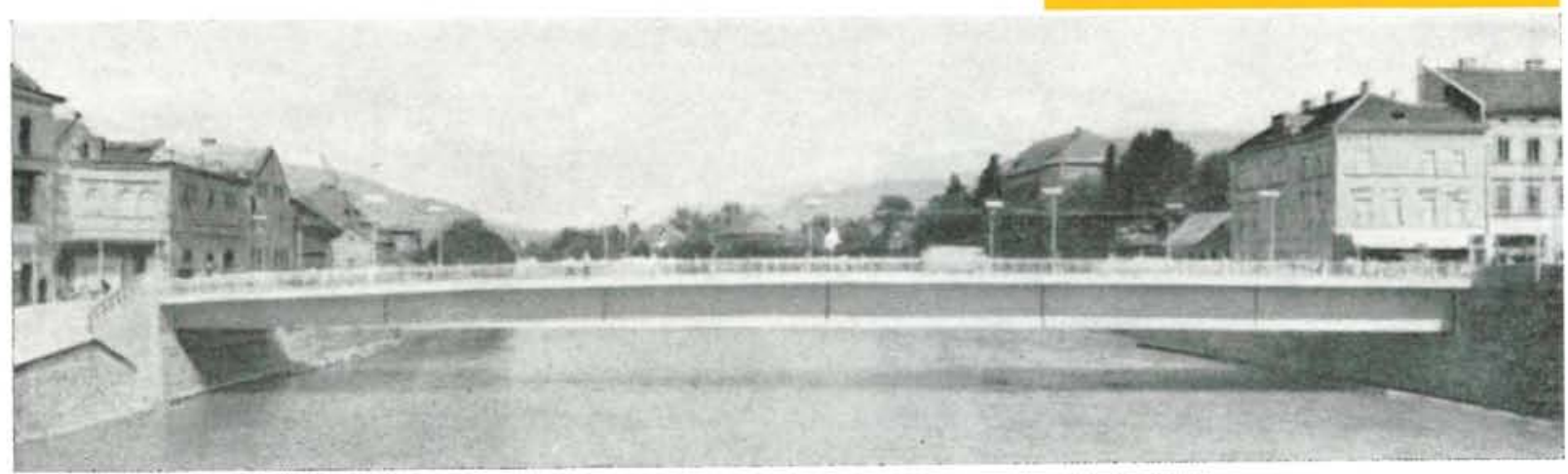




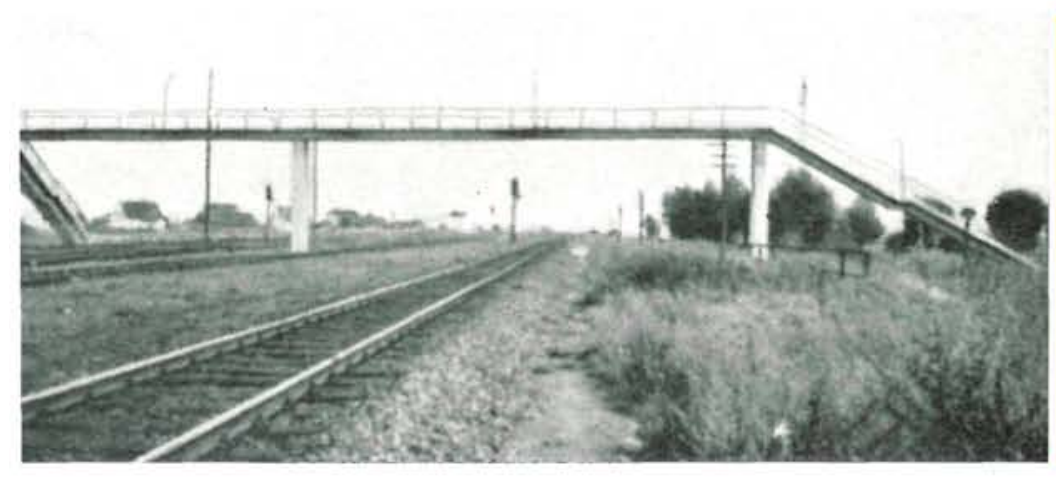

Fig. 9. Pasarela para peatones en Salzgitler-Drütte (Alemania),

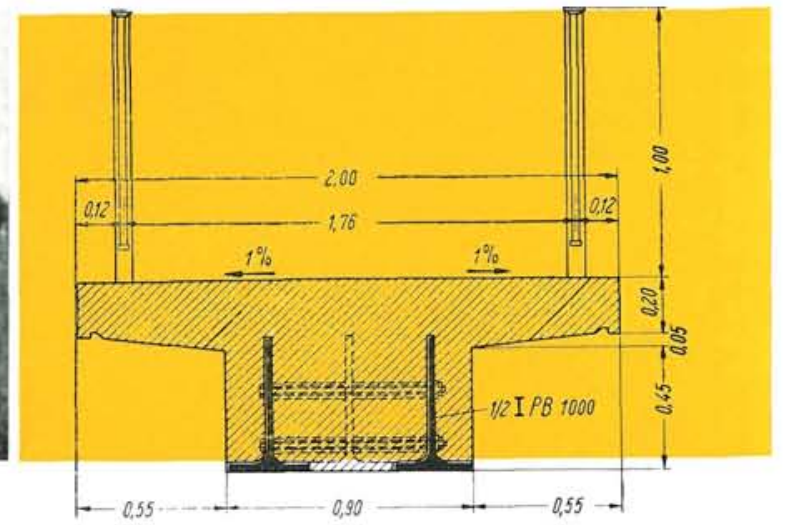

cuando su límite elástico sea alcanzado, y con pequeños incrementos de tensión sus deformaciones sean muy grandes, volverá a traspasar al hormigón la parte de solicitación que éste perezosamente le había cedido con anterioridad. Esto hace que, si bien el coeficiente de seguridad respecto al agotamiento se mantiene prácticamente invariable, permitiendo así efectuar los cálculos de pequeña importancia con gran facilidad, las tensiones y movimientos del sistema en un instante dado no sean tan fácilmente previsibles.

La retracción del hormigón al estar coartada por el acero, produce, asimismo, un estado tensional en el conjunto; como también lo hacen las diferentes inercias térmicas de los dos materiales y no, de manera apreciable, las variaciones de la temperatura ambiente, ya que sus coeficientes de dilatación son muy similares.

Todo esto lleva a que el cálculo, salvo en casos de pequeña importancia, no deba finalizar en un estudio instantáneo de la solicitación, sino que ha de ser complementado con la modificación de estos valores por el paso del tiempo.

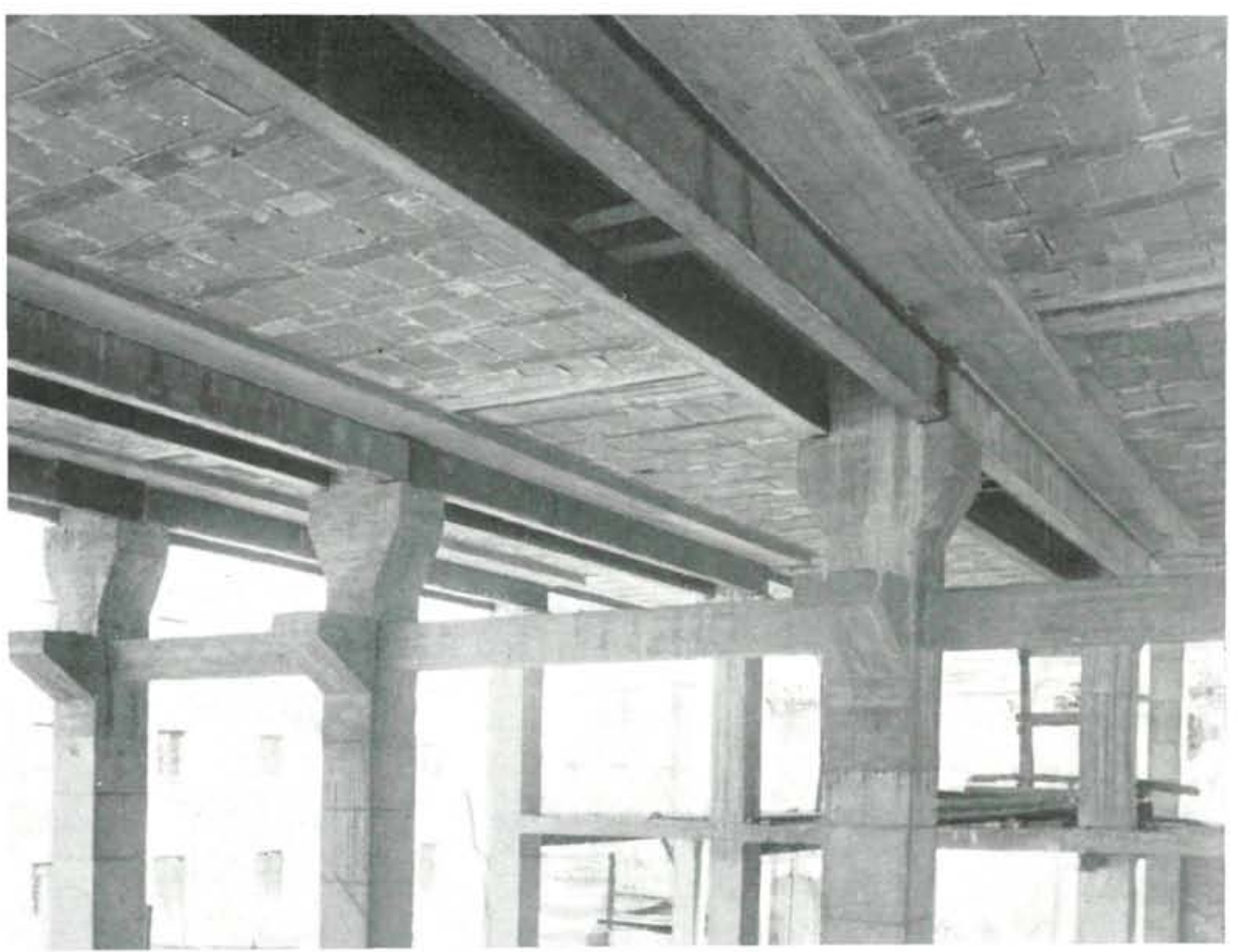

Fig. 10. Vigas mix. tas para sustentación de soportes que se interrumpen en planta baja 


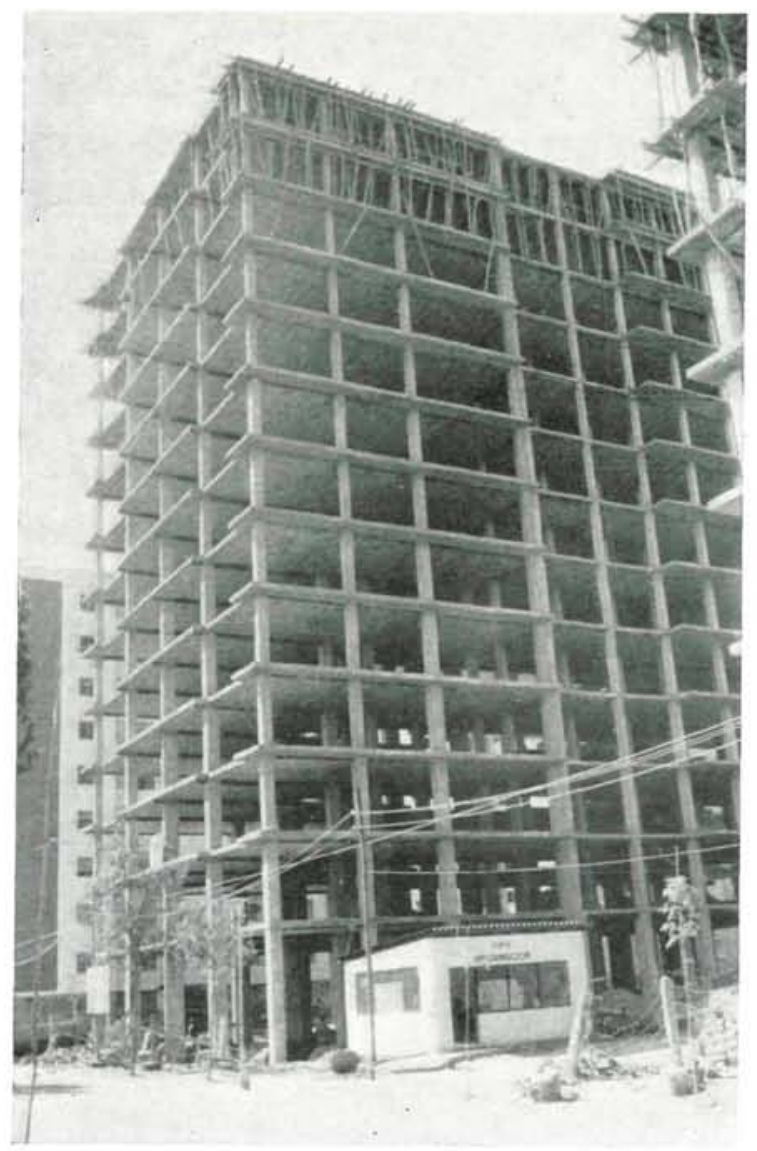

Fig. 7. Estructura mixta de edificación (España).

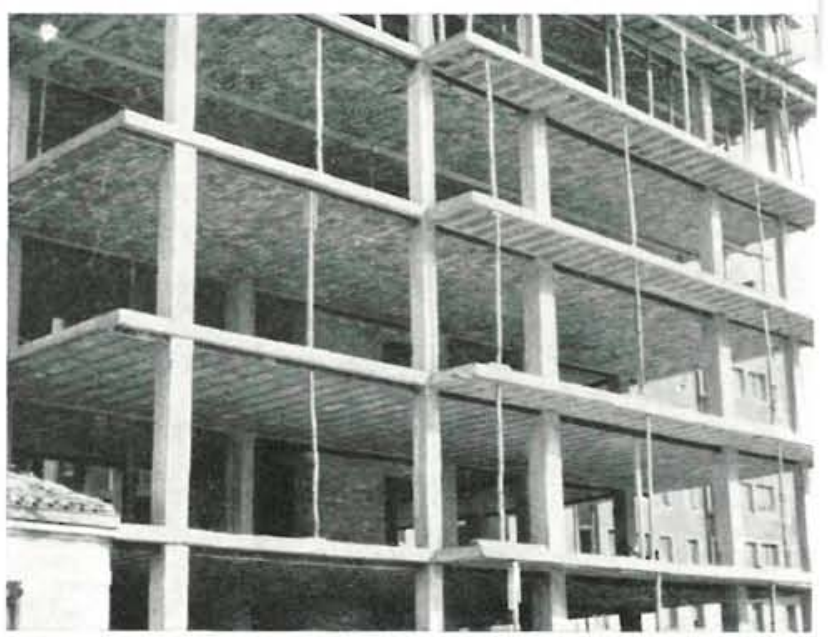

Estas ventajas aumentan aún en mayor cuantía cuando es posible introducir sistemas de presolicitación en taller o "in situ»; o cuando es posible realizar la construcción con determinados procesos constructivos.

La unión del acero y el hormigón para resistir conjuntamente las solicitaciones debidas a las cargas de servicio da lugar a que cuando se presentan acciones diferentes a estas últimas, tales como térmicas y reológicas, las particularidades de cada uno de dichos materiales influyan en el otro, $\mathrm{y}$ en algunos casos pueden llegar a condicionar el dimensionamiento.

Así, la fluencia lenta del hormigón bajo carga mantenida tiende a aumentar las deformaciones del sistema, y el acero, al no tener característica similar $(*)$, se pliega a las exigencias del conjunto aumentando sus tensiones en forma proporcional al aumento de deformación; bien es cierto que,

(*) De hecho sí existe, pero en el acero estructural su valor respecto a la del hormigón es despreciable.
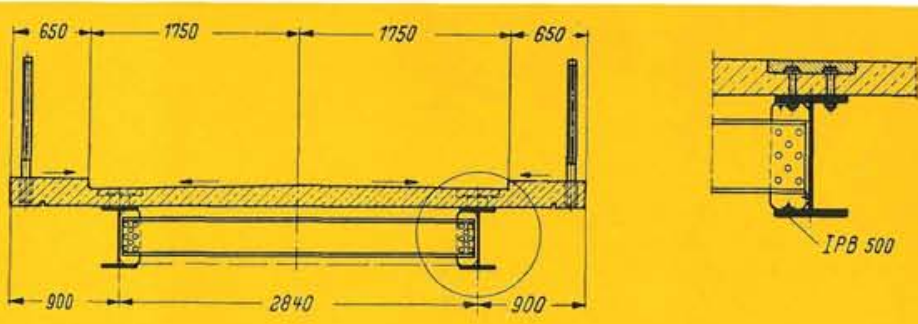

Fig. 8. Montaje de las placas prefabricadas del puente Hembergen (Alemania).

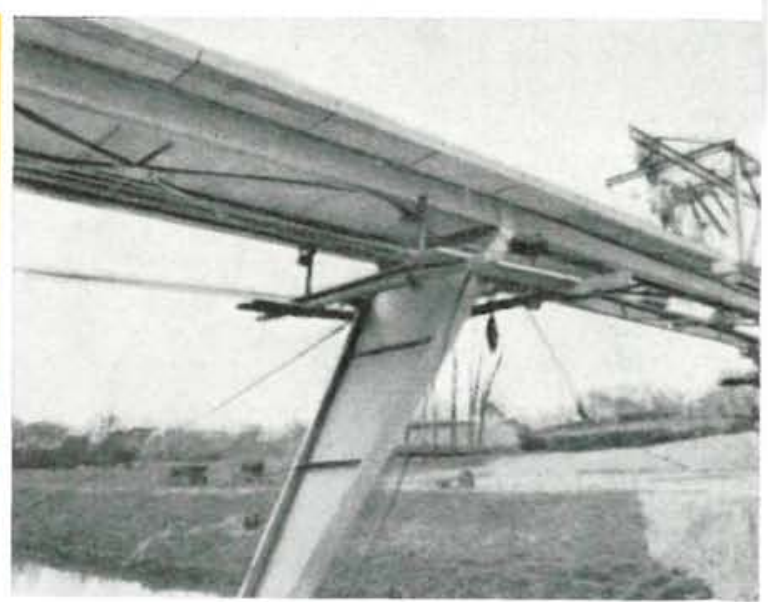



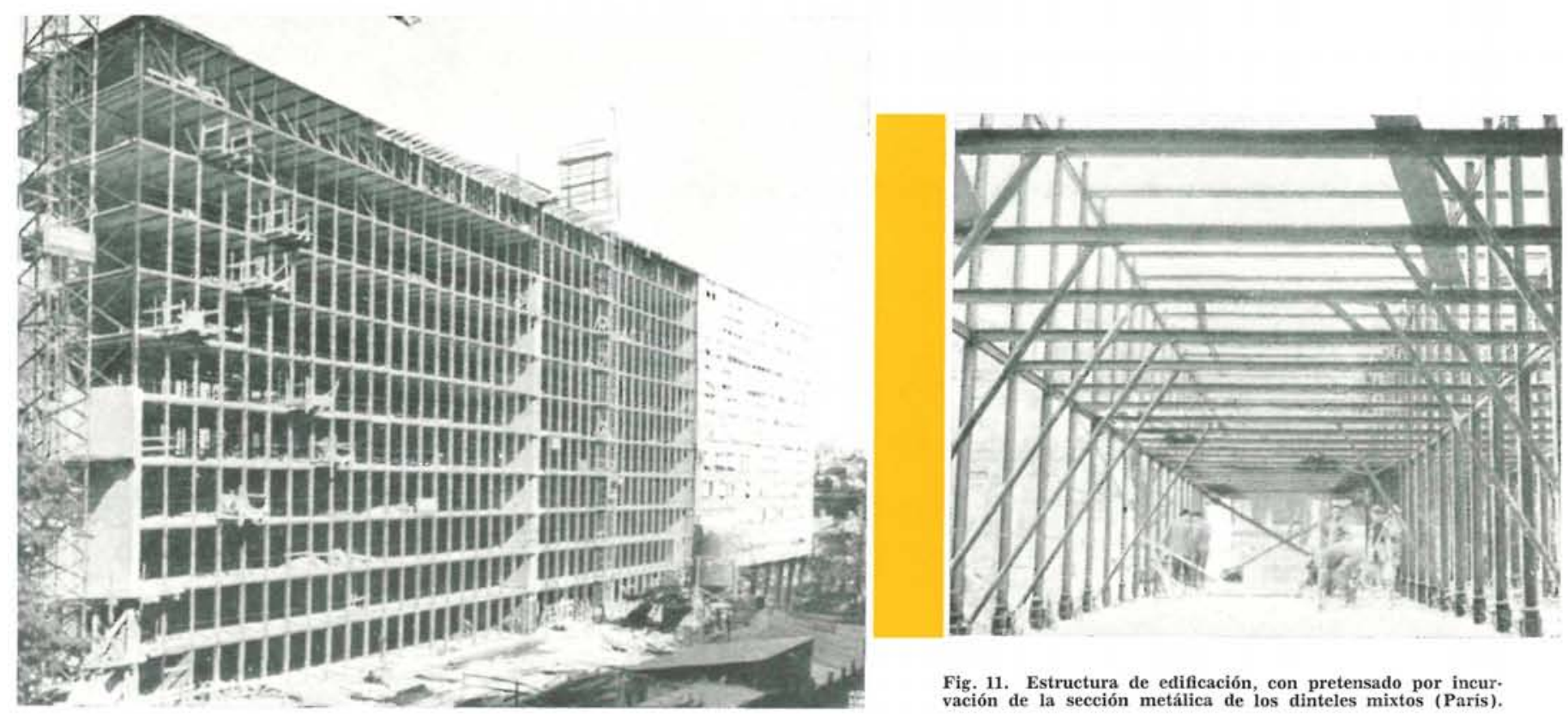

Fig. 11. Estructura de edificación, con pretensado por incur. vación de la sección metálica de los dinteles mixtos (Paris).

En el ejemplo que se describe más adelante puede apreciarse la importancia de estas modificaciones, especialmente el considerable aumento de tensión en la fibra superior del acero. Cuando la sección de hormigón de la pieza mixta es pretensada-caso de secciones con momentos negativos - es de gran interés el determinar el valor de esta variación, ya que produce en el hormigón una pérdida importante de las tensiones de compresión, que puede llegar hasta provocar la fisuración del mismo y el cambio radical del sistema resistente.

Supuesto realizados todos los cálculos antedichos con la hipótesis de trabajo conjunto de los dos materiales, es necesario garantizar que la unión exista en la realidad. En el hormigón armado y pretensado, la función de conectar los materiales corre a cargo de la adherencia, pero en el caso de piezas mixtas no es posible confiar a esta propiedad la labor de la conexión, ya que las zonas de contacto de ambos materiales son reducidas, o poseen condiciones muy desfavorables. Se hace preciso, por tanto, para igualar las deformaciones del acero y el hormigón en la fibra de contacto, estructurar unos nuevos elementos capaces de efectuar esta función: los conectadores, muy característicos de este sistema constructivo, y utilizados, asimismo, en las secciones compuestas de hormigón pretensado.

Los conectadores poseen características muy variadas y los criterios para elegir uno u otro tipo, y su consiguiente cálculo, responden a condiciones particulares de cada tipo de sección mixta, y se mostrarán con algún detalle en el segundo de los artículos previstos.

\section{Cálcullo de secciomes}

A continuación, en forma muy sucinta, se describen dos sistemas de cálculo: el uno, aproximado, válido para secciones de poca importancia, o estados iniciales de casos más complicados; y el otro, aplicable a todas las posibles secciones y en cualquier instante. Aun cuando el cálculo se establece para secciones con hormigón sin armaduras, puede ampliarse para el caso de hormigón armado o pretensado con unas sencillas consideraciones adicionales.

Esta exposición se completa con unas indicaciones sobre la forma de obtener los valores aproximados del coeficiente de fluencia y el valor de la retracción final, necesarios para el estudio completo de las secciones mixtas. 


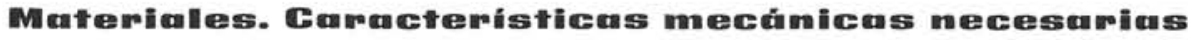

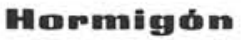

Módulo de elasticidad, $E_{b}$.

Se supone constante, es decir, independiente del tiempo y la tensión, siempre y cuando esta última no supere la considerada como admisible en el cálculo.

El valor del módulo de elasticidad puede tomarse:

$E_{b}=21.000 \sqrt{R_{k}}$, siendo $R_{k}$ la resistencia característica a 28 días.

\section{Coeficiente de fluencia.}

Su obtención puede realizarse a partir de los valores indicados por las Recomendaciones del C.E.B. (Ref. 4); sin embargo, como valor aproximado en el cálculo puede tomarse para cualquier instante el determinado por el siguiente cuadro:

\begin{tabular}{|c|c|c|c|}
\hline Forma de curado & Valor de $\varphi_{n}$ & \multicolumn{2}{|c|}{ Observaciones } \\
\hline - en agua & $0,5 K_{f}$ a $1,0 K_{f}$ & \multicolumn{2}{|c|}{ Debe aumentarse $\varphi_{n}$ en un: } \\
\hline - en aire muy húmedo & $1,5 K_{f}$ a $2,0 K_{f}$ & $10 \%$ & $20 \%$ \\
\hline - al aire libre & $2,0 K_{f}$ a $3,0 K_{t}$ & \multicolumn{2}{|c|}{ Si la dimensión mínima de pieza es: } \\
\hline - en aire muy seco & $2,5 K_{,}$a $4,0 K_{f}$ & $0,75 \mathrm{~m}$ & $1,50 \mathrm{~m}$ \\
\hline
\end{tabular}

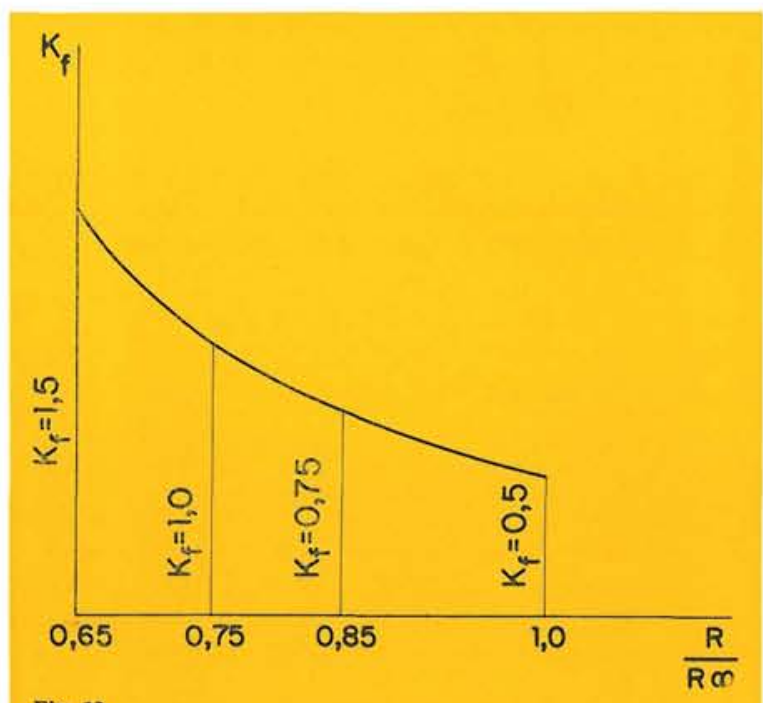

Fig. 12

(Ref. 4): Recomendaciones prácticas del C.E.B.
El valor de $K_{f}$ se obtiene de la figura 12 , en la cual $R$ es la resistencia del hormigón en el instante considerado y $R_{\infty}$ el valor teórico al cabo de tiempo infinito que, para casos prácticos, puede hacerse igual a $1,35 R_{k}$.

\section{Retracción final, $\varepsilon_{s}$.}

Como valores medios aproximados válidos para el cálculo pueden tomarse los siguientes:

- fraguado en agua $=0$

- fraguado en aire húmedo $=10 \times 10^{-5}$

- fraguado en aire $\quad=20 \times 10^{-5}$

- fraguado en aire seco $\quad=30 \times 10^{-5}$

En los casos importantes la determinación puede efectuarse por medio de los valores indicados en la referencia 4. 


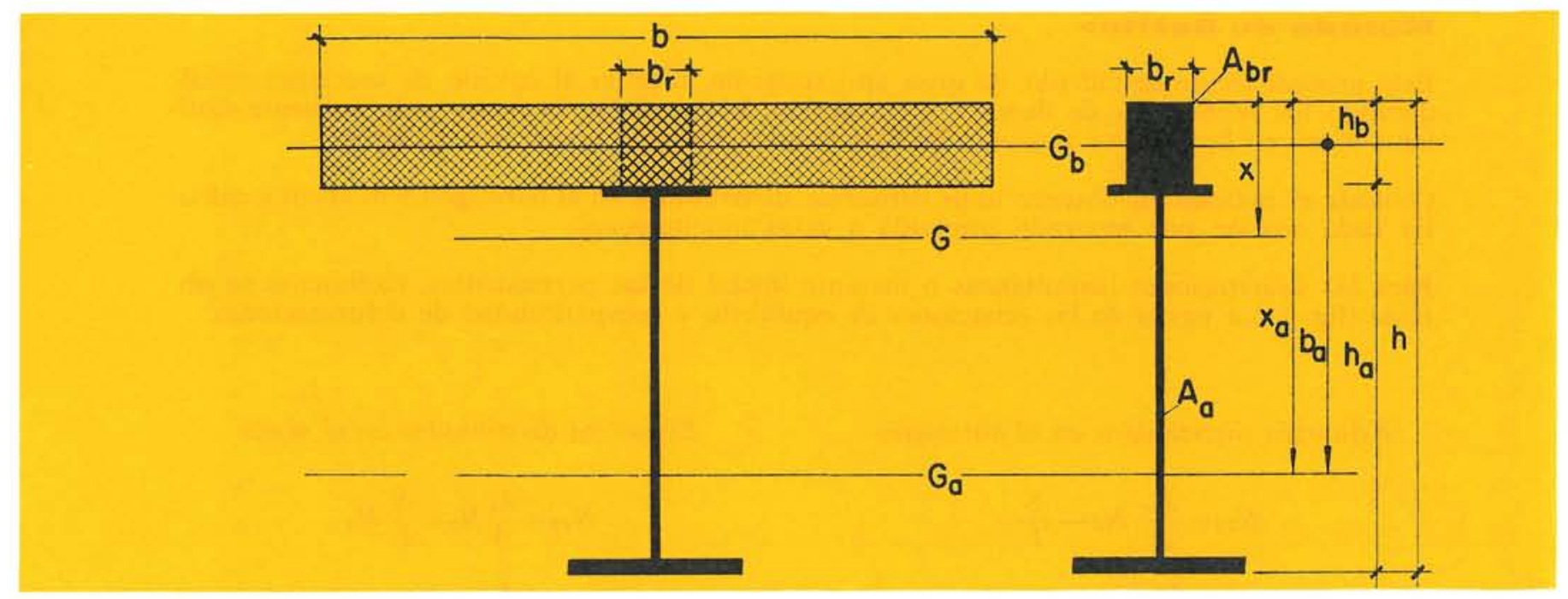

Fig. 13

\section{Bîș \\ âc cien}

La sección de hormigón se reduce a acero, dividiendo su área por el coeficiente de equivalencia $n=E_{a} / E_{b}$ (fig. 13) y tomando para $E_{b}$ los siguientes valores:

- cargas instantáneas, $E_{b}$;

- cargas permanentes, $E_{b} / 1+\varphi_{n}$.

Las características mecánicas de la sección se obtienen mediante las fórmulas siguientes, en las cuales se desprecia la colaboración del hormigón en tracción, cuando exista:

$x \leqq h_{b}$

$$
\begin{gathered}
x=\frac{A_{a}}{b_{r}}\left(\sqrt{1+\frac{2 x_{a} b_{r}}{A_{a}}}-1\right) \\
A_{b r}=x b_{r} ; \quad A=A_{b r}+A_{a} \\
b_{a}=x_{a}-\frac{x}{2} \\
S=\frac{A_{b r} A_{a}}{A} b_{a} \\
I=I_{a}+\frac{x^{3} b_{r}}{12}+b_{a} S
\end{gathered}
$$

$$
x>h_{b}
$$

$$
\begin{gathered}
x=\frac{\frac{b_{r} h_{b}{ }^{2}}{2}+x_{a} A_{a}}{h_{b} b_{r}+A_{a}} \\
A_{b r}=b_{r} h_{b} ; A=A_{b r}+A_{a} \\
b_{a}=x_{a}-\frac{h_{b}}{2} \\
S=\frac{A_{b r} A_{a}}{\mathrm{~A}} b_{a} \\
I=I_{a}+\frac{h^{3} b}{12} b_{r}+b_{a} S
\end{gathered}
$$

y los momentos resistentes de las fibras superior e inferior de la sección son:

$$
W_{\text {sup. }}=\frac{1}{x} n ; \quad W_{\text {inf. }}=\frac{I}{h-x} ;
$$

que permiten obtener los valores de las tensiones máximas en ambos materiales. 


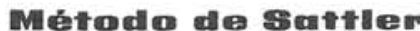

Este procedimiento de cálculo, de gran aproximación, efectúa el cálculo de secciones considerando los fenómenos de fluencia y retracción del hormigón en forma prácticamente similar a como en la realidad actúan, y ha sido confirmado por numerosos ensayos.

Consiste el método en obtener unos esfuerzos distribuidos en el hormigón y el acero y calcular cada sección por separado sometida a estas solicitaciones.

Para las solicitaciones instantáneas o instante inicial de las permanentes, fácilmente se obtiene (fig. 14) a partir de las ecuaciones de equilibrio y compatibilidad de deformaciones:

Esfuerzos distribuidos en el hormigón

$$
\begin{aligned}
N_{b, 0} & =\frac{A_{b r}}{A} N_{0}-\frac{S}{I} ; \\
M_{b, 0} & =\frac{I_{b r}}{I} M_{0} ;
\end{aligned}
$$

Esfuerzos distribuidos en el acero

$$
\begin{aligned}
N_{a, 0} & =\frac{A_{a}}{A} N_{0}+\frac{S}{I} M_{0} \\
M_{a, 0} & =\frac{I_{a}}{I} M_{0}
\end{aligned}
$$

siendo:

$$
\begin{aligned}
A_{b r} & =\frac{A_{b}}{n} \\
I_{b r} & =\frac{I_{b}}{n}
\end{aligned}
$$

y las tensiones coinciden con los valores obtenidos por el método aproximado anteriormente expuesto.

Cuando las cargas son de larga duración han de superponerse a estos esfuerzos distribuidos, otros modificativos causados por la redistribución debida a la fluencia del hormigón, al actuar sobre el mismo la solicitación $N_{b, 0}$ y $M_{b, 0}$.

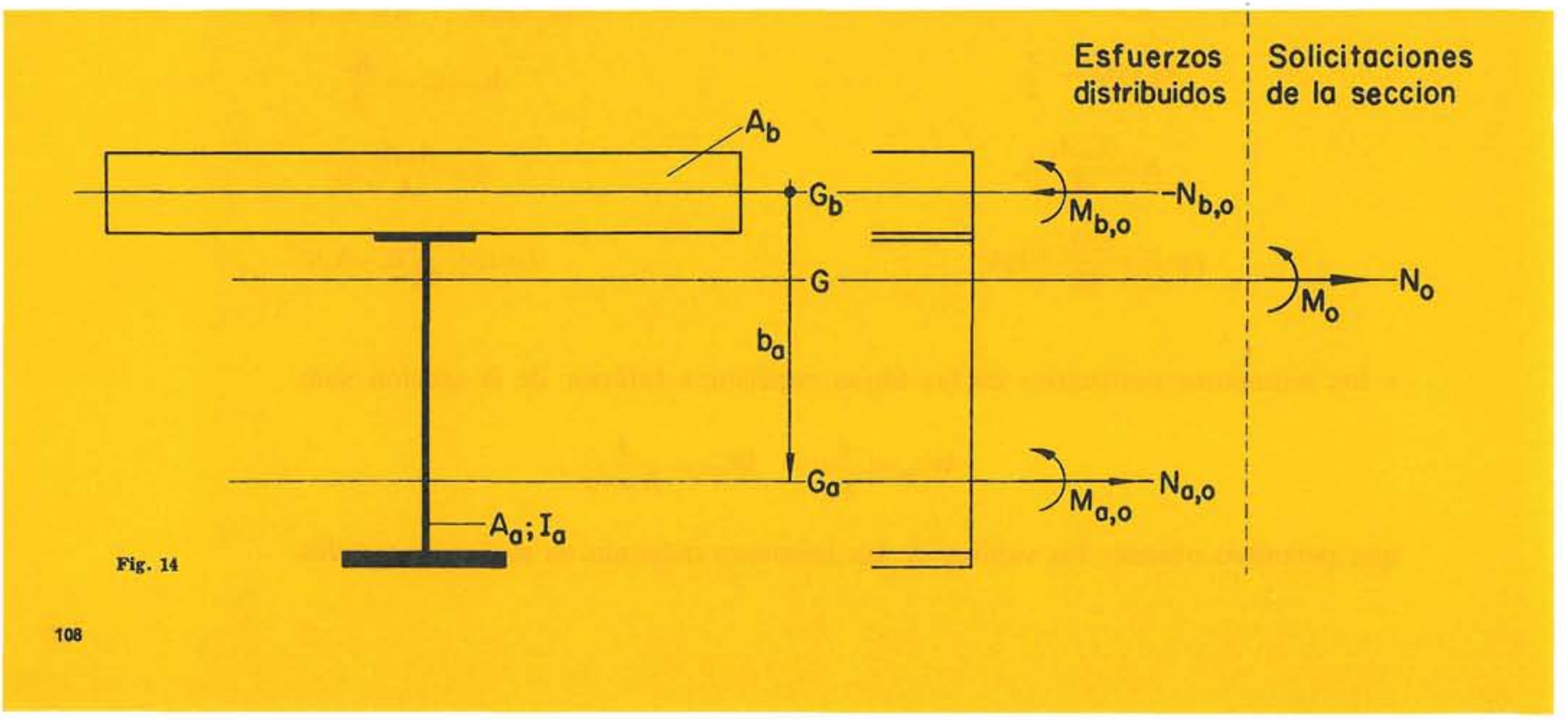


Los esfuerzos modificativos se obtienen a partir de las siguientes fórmulas:

Si $j_{a}=\frac{A_{b r} I_{b r}}{A_{a} I_{a}} \leqq 0,2$ (que suele cumplirse en secciones con $h_{a} \geqq 1 \mathrm{~m}$ )

$$
\begin{aligned}
& N_{b, n}=\left(N_{s}-N_{b, 0}\right)\left(1-e^{-\varphi_{n}}\right) \\
& N_{a, n}=-N_{b, n} \\
& M_{a, n}=b_{a} \cdot N_{b, n} \\
& M_{b, n}=-M_{b, 0}\left(1-e^{-\varphi_{n}}\right)+\frac{I_{b r}}{I_{a}} M_{a, n} \cdot H_{e}
\end{aligned}
$$

en las cuales:

$$
\begin{aligned}
N_{s} & =\frac{\varepsilon_{s}}{\varphi_{n}} E_{a} A_{b r} \\
\alpha & =\frac{A_{a} I_{a}}{A \cdot I} \\
H_{\theta} & =\frac{\alpha}{1-\alpha} \frac{e^{-\alpha \varphi_{n}}-e^{-\varphi_{n}}}{1-e^{-\alpha \varphi_{n}}}
\end{aligned}
$$

Si $j_{a}>0,2$,

$$
\begin{aligned}
& M_{a, n}=\frac{b_{a}\left(N_{s}-N_{b, 0}\right) \varphi_{n}+M_{b, 0} \frac{\varphi_{n}}{\Phi}\left(\Phi+\frac{A_{b r}}{A_{a}}\right)}{\Phi+\frac{A_{b r}}{A_{a}}+\frac{I_{b r}}{I_{a}}+\frac{A_{b r} I_{b r}}{A_{a} I_{a}} \frac{1}{\Phi}+b_{a}{ }^{2} \frac{A_{b r}}{I_{a}}} \\
& M_{b, n}=\left(-M_{b, 0} \cdot \varphi_{n}+M_{a, n} \cdot \frac{I_{b r}}{I_{a}}\right) \frac{1}{\Phi} \\
& N_{a, n}=-\frac{1}{b_{a}}\left(M_{a, n}+M_{b, n}\right) \\
& N_{b, n}=-N_{a, n}
\end{aligned}
$$

siendo: $\Phi=1+0,65 \varphi_{n}$.

La laboriosidad de estos cálculos, más aún, el tener que efectuar diversos tanteos para llegar a un dimensionamiento ajustado, hizo pensar en la obtención de un programa de cálculo electrónico para cualquier tipo de secciones mixtas, y la ejecución de tablas de las características mecánicas de secciones mixtas sencillas utilizadas de manera continua en la práctica. Esto llevó por un lado a la obtención del programa (ficha E-34) que figura al final de esta revista en una de las fichas de cálculo electrónico, en la que se pueden observar algunas de las múltiples posibilidades de construcción de este tipo de piezas; y por el otro, a una serie de tablas que figuran en el anejo de la obra en preparación antes mencionada $\left({ }^{*}\right)$.

(*) Para perfiles de tipo europeo pueden utilizarse las tablas que figuran en la referencia 5.

(Ref. 5): Querschnitt-Verbundträger im Hochbau (Sections mixtes dans le bâtiment). 


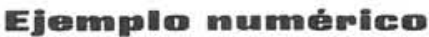

Para aclarar todos estos puntos, se expone a continuación un ejemplo numérico resuelto con la ayuda de estas tablas (se incluye la tabla precisa para este cálculo) y mediante el programa basado en el método de Sattler.

La sección considerada se indica en la figura 15. Las características de los materiales son:

$$
\begin{aligned}
& R=360 \mathrm{~kg} / \mathrm{cm}^{2} \\
& E_{b}=390.000 \mathrm{~kg} / \mathrm{cm}^{2} ; \quad \varphi_{n}=2,5 ; \quad \varepsilon_{s}=20 \times 10^{-5} ; \\
& E_{a}=2.100 .000 \mathrm{~kg} / \mathrm{cm}^{2} ; \quad n_{0}=\frac{E_{a}}{E_{b}}=5,384 ; \\
& n_{n}=\frac{\left(1+\varphi_{n}\right) E_{a}}{E_{b}}=18,844 \\
& b_{\text {ro }}=27,86 \mathrm{~cm} \text {; } \\
& b_{m}=7,96 \mathrm{~cm}
\end{aligned}
$$

y de la tabla adjunta se obtienen, por interpolación:

$$
\text { para } \begin{aligned}
t= & 0: \\
x & =17,9 \mathrm{~cm} ; \\
A & =596 \mathrm{~cm}^{2} ; \\
I & =203.413 \mathrm{~cm}^{4} ; \\
\frac{W_{\text {sup. }}}{n} & =11.408 ; W_{\text {sup. }}=61.421 \mathrm{~cm}^{3}: \\
W_{\text {inf. }} & =5.197 \mathrm{~cm}^{3} ; \\
Z & =47,2 \mathrm{~kg} / \mathrm{cm} ;
\end{aligned}
$$

$$
\text { para } \begin{aligned}
t= & n: \\
x & =28,25 \mathrm{~cm} ; \\
A & =297,26 \mathrm{~cm}^{2} ; \\
I & =133.976 \mathrm{~cm}^{4} ; \\
\frac{W_{\text {sup. }}}{n} & =4.757 ; W_{\text {sup. }}=89.641 \mathrm{~cm}^{3} ; \\
W_{\text {inf. }} & =4.657 \mathrm{~cm}^{3} ; \\
Z & =54,5 \mathrm{~kg} / \mathrm{cm} ;
\end{aligned}
$$

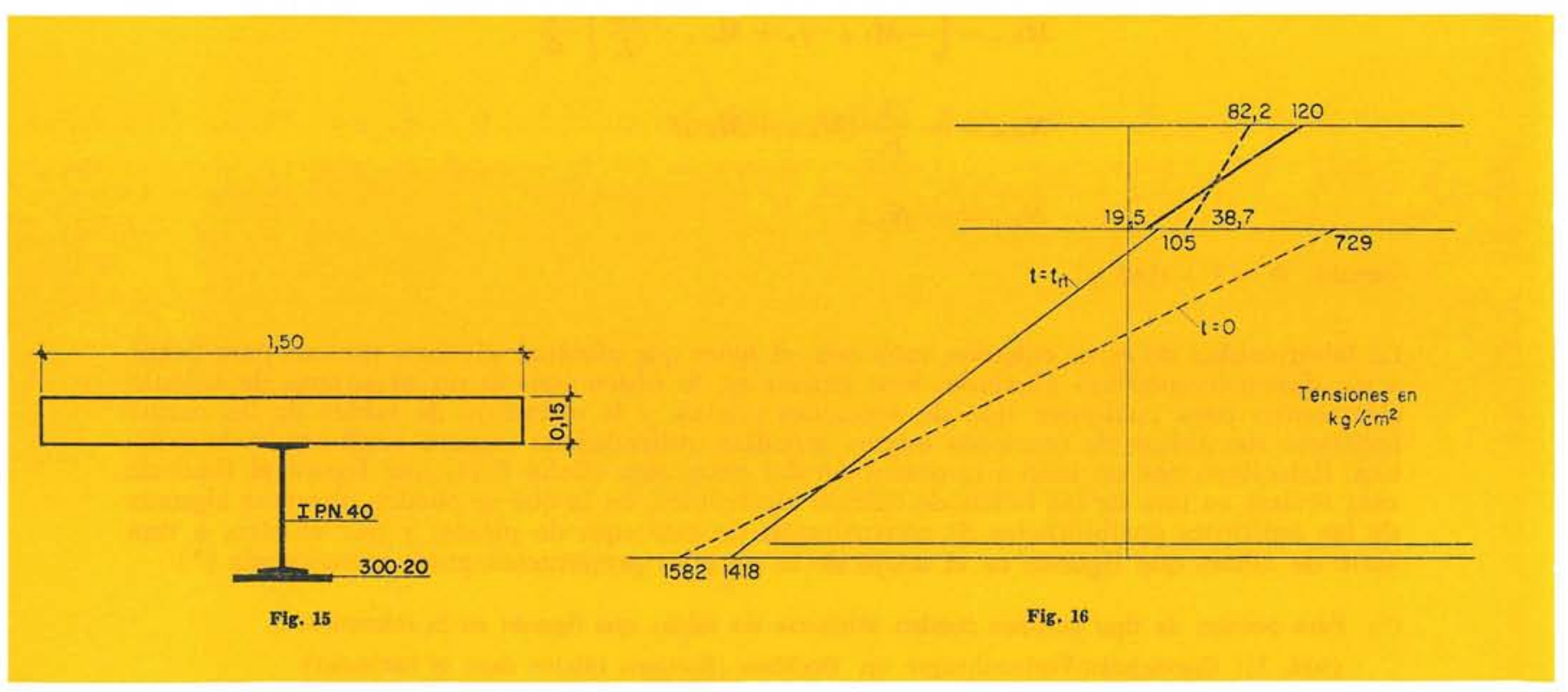




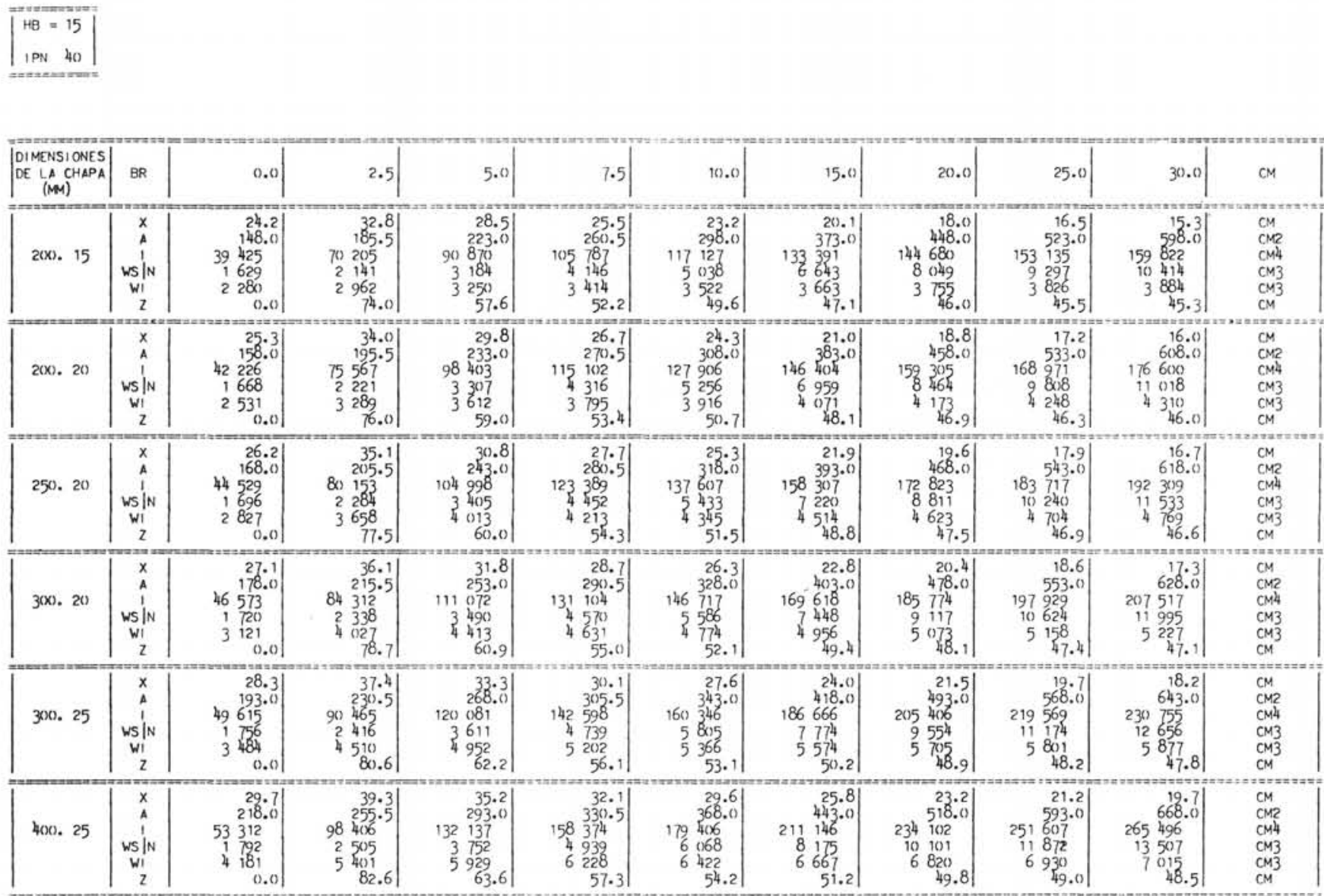

Para un momento exterior permanente $M_{0}=73,705 \mathrm{~m} \cdot \mathrm{t}$, las tensiones en ambos instantes, inicial y definitivo, se muestran en la figura 16; mientras que los valores proporcionados por el programa electrónico son:

\section{CARACTERISTICAS DE LA SECCION DE HORMIGON}

$\begin{array}{lr}X B= & 7.50 \mathrm{CM} \\ A B= & 2250.00 \mathrm{CM} 2 \\ A B R= & 417.86 \mathrm{CM} 2 \\ I B= & 42187.50 \mathrm{CM4} \\ I B R= & 7834.82 \mathrm{CM4}\end{array}$

CARACTERISTICAS DE LA SECCION DE ACERO ESTRUCTURAL

$\begin{array}{lrl}A E & = & 179.46 \mathrm{CM} 2 \\ V E & = & 14.98 \mathrm{CM} \\ I E & = & 4747.05 \mathrm{CM} 4\end{array}$

CARACTERISTICAS DE LA SECCION MIXTA

$\begin{array}{rrr}B Y= & 10.37 \mathrm{CM} \\ A= & 597.32 \mathrm{CM} 2 \\ S= & 4333.91 \mathrm{CM} 3 \\ 1= & 204882.23 \mathrm{CM} 4\end{array}$

TENSIONES TOTALES EN LOS MATERIALES. INSTANTE $T=0$

$\begin{array}{rrrr}T \text { BO }= & -119.40 \mathrm{kG} / \mathrm{CM} 2 \\ T \text { BU }= & -19.19 \mathrm{KG} / \mathrm{CM} 2 \\ T E O O= & -103.31 \mathrm{kG} / \mathrm{CM} 2 \\ T E U O= & 1407.61 \mathrm{KG} / \mathrm{CM} 2\end{array}$

Puede observarse que las diferencias sólo son apreciables en los valores de las tensiones en el hormigón en el instante final, siendo el resto de los resultados prácticamente semejantes.
TENSIONES TOTALES EN LOS MATERIAI.ES. INSTANTE T=N

$\begin{aligned} T B O= & -72.79 \mathrm{KG} / \mathrm{CM} 2 \\ T \mathrm{BU}= & -47.03 \mathrm{KG} / \mathrm{CM} 2 \\ T \mathrm{EO}= & -755.21 \mathrm{KG} / \mathrm{CM} 2 \\ T \mathrm{TU}= & 1586.20 \mathrm{KG} / \mathrm{CM} 2\end{aligned}$




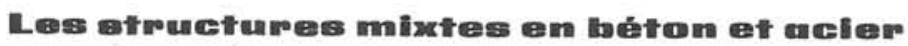

\section{J. Martínez Calzón, ingénieur des Ponts et Chaussées}

Cet article met en relief les principales causes pour lesquelles les structures mixtes sont peu employées dans la construction espagnole actuelle, ainsi que la possibilité qu'a cette technique, dans un futur très proche, d'être utilisée sans réserves dans les cas où son emploi serait indiqué.

Sont présentées très succinctement une méthode simple approximative et une autre plus précise due à Sattler pour le calcul de sections. L'article montre également, à l'aide d'un exemple, l'utilisation de quelques tables et d'un programme de calcul électronique qui doivent figurer dans un ouvrage de l'auteur de cet article, qui paraitra prochainement, visant à éviter les difficultés des calculs et à aider au dimensionnement de ce type de structures.

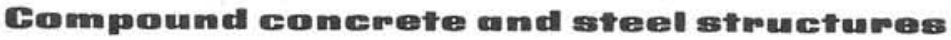

J. Martínez Calzón, civil engineer

The reason is given why compound structures are not more widely used in Spanish constructional work at present. The possibility is discussed that this technique shall be used without inhibition in the near future, in all those cases where it is the best constructional solution.

A succint account is given of a simple, approximate method, and also a more accurate one, due to Sattler, to calculate compound steel and concrete sections. By means of an example, it is shown how to make use of tables, and of an electronic program, which are to be included in a book shortly to be published, written by the author of this article. These tables and electronic program tend to avoid the laboriousness of calculations and are an aid in designing this type of structures.

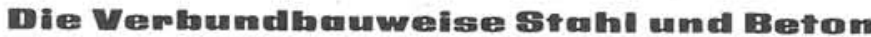

\section{J. Martínez Calzón, Bauingenieur}

Die Verbundbauweise Stahl und Beton sind im gegenwärtigen spanischen Bau wenig verwendet, aber es ist möglich, dass diese Technik in nächster Zukunft anwenden werden, in den Fällen, in den ihre Anwendung geeignet sei.

Kurzgefasst zeigt man eine einfache annähernde Methode und eine andere genauer von Sattler für die Berechnung von Querschnitte an. Man zeigt auch durch ein Beispiel die Anwendung von einen Tabellen und einem Programm elektronischer Berechnung an, die sich in einem Werke von nächster Erscheinung vom Verfasser dieses Artikels verzeichnet finden werden. Damit wird man die Schwiriegkeiten von Berechnungen vermeiden und die Dimensionierung dieser Verbundbau wird leichter sein. 